\title{
The Effect of an Eight-Week Rope Skipping Exercise Program on Interleukin-10 and C-Reactive Protein in Overweight and Obese Adolescents
}

\author{
Iman Zakavi ${ }^{1,} ;$ Banafshe Bizhani $^{2}$; Mojgan Bani Hashemi ${ }^{3}$; Emam Ghaisii ${ }^{3}$ \\ ${ }_{1}^{1}$ Department of Sports Physiology, Abadan Medical Sciences School, Abadan, IR Iran \\ ${ }^{2}$ Department of Statistics, Ahvaz Jundishapur University of Medical Sciences, Ahvaz, IR Iran \\ ${ }^{3}$ Department of Physical Education and Sport Sciences, Islamic Azad University, Khorasgan (Isfahan) Branch, Isfahan, IR Iran \\ *Corresponding author: Iman Zakavi, Department of Sports Physiology, Abadan Medical Sciences School, Abadan, IR Iran. Tel: +98-9168143051, E-mail: imanzakavi@yahoo.com \\ Received: October 22, 2014; Revised: May 9, 2015; Accepted: May 10, 2015 \\ $\mathrm{aC}$
}

Background: The different effects of exercise on obesity in obese adolescents have not sufficiently been studied.

Objectives: This study aimed to investigate the effect of an eight-week rope skipping exercise on interleukin-10 (IL-10) and C-reactive protein (CRP) in obese and overweight adolescents.

Patients and Methods: In this semi-experimental study, using purposive convenience sampling 30 overweight and obese teens were randomly divided into two groups: the experimental (height $165.28 \mathrm{~cm}$; weight $85.53 \mathrm{~kg}$ and age 13.73 years old) and control (height $164.54 \mathrm{~cm}$; weight $83.02 \mathrm{~kg}$ and age 13.93 years old) groups. Then the experimental group performed the eight-week rope skipping exercise program while the control group did not receive any intervention and was only following up. Before and after the exercise, the variables including weight, fat percentage, body mass index (BMI) and the maximum oxygen consumption (Vo2max) in both groups were measured. To assess the amount of serum IL-10, CRP, 48 hours before and after the exercise, fasting blood samples were taken during the two-stage mode. The correlated t-test and the independent t-test were used to compare the intragroup and intergroup relationships, respectively.

Results: There was no significant change in the serum levels of IL-10 ( $\mathrm{P}>0.05)$; however, the intragroup comparison in the experimental group showed a significant increase in serum levels of IL.10 $(\mathrm{P}<0.05)$. Moreover, the variables of the weight, BMI, fat percentage, V02max and CRP were significantly changed $(\mathrm{P}<0.05)$.

Conclusions: A rope skipping protocol increases the anti-inflammatory index, reduces the risk of cardiovascular disease, and improves the body compounds and immune system of the obese and overweight teens.

Keywords: Exercise; IL-10; CRP; Overweight; Obesity

\section{Background}

Obesity is now one of the world's health problems, and its prevalence is spreading. There is an opinion that the cause of obesity is really complex and ambiguous and is not well-known (1). Obesity and overweight among children and adolescents is notable and there is a specific experience of overweight among children and adolescents in all countries of the world and maybe also continued after puberty (2). Obesity is a risk to create a certain types of infections, inflammatory disorders and cardiovascular diseases (3). It is known that low mobility is largely involved in creating the obesity and infection (4). This issue is properly proven that the risk factors for cardiovascular diseases in childhood and adolescence can predict the risk of the disease in adults (5). Therefore, a lower age is a good opportunity to create a healthy lifestyle without the emergence of infectious agents and diseases in the future (2).

By reducing the inflammation, clotting, obesity markers and increasing anti-inflammatory marker the physi- cal activity can reduce the death rate in people exposed to the risk (6). As even some of the studies consider nonphysical activity as independent of obesity, and associated with an increased inflammatory marker (7). Due to increased gene expression of cytokines, obesity is closely associated with large quantities of inflammation and since the regular exercise reduces inflammation (8), as well as strengthens and improves the functioning of the immune system (9), regular exercise also somewhat causes weight loss that in turn it reduces levels of inflammatory symptoms (4). Cytokines are proinflammatory hormones that are involved in regulating the growth and differentiating the function of most of the body cells. They also play an important role in strengthening the immune response (10). Among the cytokines, interleukin-10 (IL-10) is secreted by monocytes, macrophages and lymphocytes, and considered as a beneficial and protective cytokine in human metabolism (11). Interleukin-10 protects the excessive proinflammatory response that

Copyright (C) 2015, Ahvaz Jundishapur University of Medical Sciences. This is an open-access article distributed under the terms of the Creative Commons Attribution-NonCommercial 4.0 International License (http://creativecommons.org/licenses/by-nc/4.0/) which permits copy and redistribute the material just in noncommercial usages, provided the original work is properly cited. 
is more in the obese subjects (12). Because obesity is considered as a proinflammatory state, serum multiple cytokine levels may vary according to the degree of weight loss in obese patients. The IL-10 levels increase due to the weight loss in obese patients along with improved metabolic disorders (11). Recent studies have demonstrated that the IL-10 level prior to exercise is lower compared to the post-exercise level; so, regular exercise with moderate intensity reduces proinflammatory cytokines such as IL- 6 and increases anti-inflammatory cytokine such as IL-10 (12). Several studies have shown the inverse relationship between fitness and the amount of lymphocytes and inflammatory markers such as IL-6 and C-reactive protein (CRP), whereas IL-10 has been shown to be positively correlated with fitness. Encouraging the obese individuals, especially children and teens who face with a critical period of their life, to participate in a regular and a desirable sports activity forces the researchers to encourage the people in sports as well as guarantee their health $(13,14)$.

The CRP has been identified as a risk factor for cardiovascular disease (14). The risk of heart attack in people with the CRP concentration higher than $2.11 \mathrm{mg} / \mathrm{L}$ is three times higher than those who will not have levels higher than $55.0 \mathrm{~g} / \mathrm{L}$ (15). Research results in this field can be inconsistent, as some studies have shown no change in the CRP level regarding the effect of aerobic exercise on obesity (16) and others have reported a decrease in CRP regarding the effect of exercise (17).

Therefore, according to the contradiction in the research results, it seems that there is no general consensus on the role of exercise (and type of exercise) in the IL-10 and CRP, yet, and no research has been conducted on the effect of the rope skipping exercise protocol over IL-10 and CRP in the obese adolescents; so, an incentive was created to conduct a research in this area and about the effect of eight weeks of rope skipping exercise on the IL-10 and CRP in obese adolescents.

\section{Objectives}

This study aimed to investigate the effect of an eightweek rope skipping exercise on IL-10 and CRP in obese and overweight adolescents.

\section{Patients and Methods}

In terms of the method, the present study was semi-experimental; according to the length of time it was a crosssectional and field study; and in terms of the use of the results, it was an applied study. The participants of this study were all male students in middle and high schools in Baghmalek City and were selected using the purposive convenience sampling. From a total of 2,095 students in the study population, 30 students with a body mass index (BMI) higher than 25 and 30 participated in the study and were divided into two groups of overweight and obese teens (experimental and control). After the announcement of the public recall, the measurements of height, weight and BMI were done. To choose the healthy obese persons having the ability to participate in exercises, the physical activity readiness questionnaire (PAR-Q) was used. After specifying the status of the obesity among the people with the mentioned conditions by a convenience random sampling method, 30 people were randomly selected and divided into two equal groups: the experimental and control ones. Subjects who had a history of the sport and disease as well as subjects who were using a method of weight loss were excluded from the study. All participants received the required information on the research and after reading, subjects and their parents were asked, if they wish to participate in the study, to sign a written consent. Physical features of the experimental and control groups are shown in Table 1.

All variables, including age (years), and height $(\mathrm{cm})$ were measured using a SECA height gauge (Germany) with a sensitivity of $1 \mathrm{~mm}$. A digital laboratory scale (made in Iran) with a sensitivity of $1 \mathrm{mg}$ was used for weighing subjects. Using the Caliper Longo models with a precision of $1 \mathrm{~mm}$ (made in Germany) body fat percentage was measured from two points of the body (triceps brachii and leg). Then, by putting a total of two points measured in $\mathrm{mm}$ and putting it in Slaughter et al.'s equation, the fat percentage rate was obtained (Equation 1) (6).

Equation 1.

Percentage of body fat $=(1+($ Total thickness of three skin head brachii and leg) $\times 0.735$ )

Subjects performed a ROCKPORT 1-mile walk test as aerobic performance. By using a ROCKPORT 1-mile walk test, the peak aerobic power (VO2max) of subjects was calculated by the equation 2 (18):

Equation 2.

Peak aerobic power $=(132.853-0.7690 \times$ weight $)-(0.3877$ $\times$ age $)+1 \times 6.315-(3.2649 \times$ time $)-(0.1565) \times$ heart rate $)$

Weight in pounds; time: minutes and hundredths of seconds; age:year; sex male: one, peak aerobic power: $\mathrm{mL} /$ $\mathrm{kg} / \mathrm{min}$.

The ELISA method and Bionic kit (made in Iran) were used to measure the CRP.

Using a human IL-10 kit, ELISA kit, (the BOSTER Co. USA) with specification of Cat No: EK0864, the sensitivity of 1 pg per $1 \mathrm{ml}$ and intra-and inter-assay coefficients of variation of the level of IL-10 were measured as CV $<12 \%$ and CV $<10 \%$, respectively.

In the experimental group, the exercise program includes exercise of skipping with ropes. The eight-week rope skipping exercise program is shown in Table 2. Warming-up and stretching for five minutes and cooling

\begin{tabular}{lcc}
\hline \multicolumn{3}{l}{ Table 1. Demographic Profile of the Participants ${ }^{\mathrm{a}}$} \\
\hline Groups & Age, $\mathbf{y}$ & Height, cm \\
\hline Experimental & 13.73 & 165.28 \\
Control & 13.93 & 164.5 \\
\hline
\end{tabular}

\footnotetext{
${ }^{\mathrm{a}} \mathrm{N}=15$.
} 
down for five minutes were used. The exercise time was 30 minutes per session. Time for the sets was increased per week, and in the fourth, seventh, eighth weeks, in addition to increasing the time of sets in any session, the number of jumps was also increasing.

In all periods of exercise, the fatigue and the feeling of warmth were as two alarms for stopping the practice. If there was a feeling of warmth, the cold packs were used to reduce the temperature. Progressive training was conducted and all the times and the jumps were recorded. Exercise was done for five times a week. The protocol used was adapted from a study by Kim et al. (2), which assessed the effect of rope exercises on obese and lean boy teens; furthermore, the protocol with this intensity not only did not lead to any complications for obese and thin teens, but also led to improved body composition, and some of the inflammatory indexes in obese kids (2). For this reason, in the present study the roping exercises, with an intensity expressed in Kim et al. (2), was carried out in overweight and obese adolescents for eight weeks. After justifying the subjects, they were asked to follow normal sleep patterns and diet during the study before running the tests. Blood samples were taken from the anterior vein of the left hand of the subjects in a sitting state just 48 hours before the start of the first session (pretest) and 48 hours after the last exercise session (end of the eighth week), after overnight fasting and resting, 8 am and every time with a value of $5 \mathrm{cc}$. Blood sample taken was incubated at room temperature and then serum was separated from a blood clot by centrifugation (3000 RPM for 10 minutes), and was stored in freeze $70^{\circ} \mathrm{C}$ until the time of the measurement. After collecting blood samples at posttest, all blood samples were removed from a freezer in one day and the target testing was conducted on the basis of the relevant protocols. On both occasions of blood sample collection the subjects were overnight fasting at least for 12 hours. Also, the experimental subjects were asked to do not have any physical activity or the long walk for 48 hours after the end of the exercise period. All operations for collecting blood samples were performed by a laboratory technician at the university laboratory of immunology. After eight weeks of exercise, once again height and weight were measured to determine BMI and body fat percentage and VO2max. The Kolmogorov-Smirnov test was used to test the normality of the data. The t-test was used for intragroup comparison and t-test was used for intergroup comparison. All statistical calculations were performed using SPSS version 19. The results of the statistical analysis were completely reviewed in the results section of the study.

\section{Results}

Physical characteristics of the subjects in the experimental and control groups are shown in Table 3. Table 3 shows the change of variables between the groups. The intergroup (independent t-test) and intragroup (paired t-test) comparisons indicated that the rope skipping exercises caused a significant difference in amounts of weight, body fat percentage, BMI, VO2max, and serum CRP $(\mathrm{P}>0.05)$. The comparison between groups (independent t-test) showed that serum IL-10 levels did not change significantly ( $\mathrm{P}>0.05)$, but the comparison within groups (paired t-test) showed that the serum levels of IL-10 increased significantly in the experimental group (P $>0.05)$. Figure 1 shows a comparison between the mean change in serum CRP $(\mathrm{mg} / \mathrm{L})$ in the experimental (pretest: $3.90 \pm 1.4$, posttest: $1.97 \pm 0.84$ ) and control (pretest: 4.33 \pm 2.37 , posttest: $4.76 \pm 2.137$ ) groups after eight weeks of the rope skipping exercises and in a comparison between and within groups there was a significant reduction $(\mathrm{P}=$ $0.000)$. Figure 2 shows a comparison between the mean change in serum IL-10 levels $(\mathrm{pg} / \mathrm{mL})$ in the experimental (pretest: $23.92 \pm 11.36$, posttest: $33.91 \pm 22.91$ ) and control (pretest: $21.17 \pm 10.11$, posttest: $21.84 \pm 21.93$ ) groups after eight weeks of the rope skipping exercises, and in a comparison between groups there was not significant ( $\mathrm{P}=$ 0.207 ), but in a comparison within the groups, the experimental group had a significant increase $(\mathrm{P}=0.014)$.

\begin{tabular}{lcc}
\hline Table 2. An Eight-Week Program of Rope Training a,b & \\
\hline Week & The Time of Jump in Each Set, min & Number of Jumps per Minute \\
\hline I & 1 & 60 \\
II & 1.5 & 60 \\
III & 2 & 60 \\
IV & 2.5 & 90 \\
V & 3 & 90 \\
VI & 3.5 & 90 \\
VII & 4 & 110 \\
VIII & 4.5 & 120 \\
\hline
\end{tabular}

\footnotetext{
a Duration of exercises per session are 30 minutes.

b Rest time between sets is 30 seconds.
} 
Zakavi I et al.

\begin{tabular}{|c|c|c|c|c|}
\hline Statistical Indicators & Pretest & Posttest & T Independent & P Value \\
\hline Weight, kg & & & -6.70 & 0.00 \\
\hline Control & $83.02 \pm 15.45$ & $83.23 \pm 15.49$ & & \\
\hline Experimental & $85.53 \pm 15.59$ & $85.53 \pm 15.28$ & & \\
\hline Fat Percentage, $\%$ & & & -8.777 & 0.00 \\
\hline Control & $35.06 \pm 2.456$ & $35.532 \pm 2.405$ & & \\
\hline Experimental & $34.74 \pm 2.71$ & $32.32 \pm 2.61$ & & \\
\hline BMI, $\mathrm{kg} / \mathrm{m}^{2}$ & & & -9.48 & 0.00 \\
\hline Control & $30.404 \pm 3.198$ & $30.478 \pm 3.203$ & & \\
\hline Experimental & $31.046 \pm 3.55$ & $29.14 \pm 3.41$ & & \\
\hline VO2MAX, mL/kg/min & & & 2.672 & 0.012 \\
\hline Control & $25.56 \pm 1.33$ & $24.76 \pm 2.824$ & & \\
\hline Experimental & $26.92 \pm 4.62$ & $30.08 \pm 1.37$ & & \\
\hline IL-10, pg/mL & & & 1.292 & 0.207 \\
\hline Control & $21.17 \pm 10.11$ & $21.84 \pm 21.93$ & & \\
\hline Experimental & $23.92 \pm 11.36$ & $33.91 \pm 22.91$ & & \\
\hline CRP, mg/L & & & -7.500 & 0.00 \\
\hline Control & $4.33 \pm 2.37$ & $4.76 \pm 2.137$ & & \\
\hline Experimental & $3.90 \pm 1.4$ & $1.97 \pm 0.84$ & & \\
\hline
\end{tabular}

Abbreviations: BMI, body mass ind
b Data are presented as mean \pm SD.

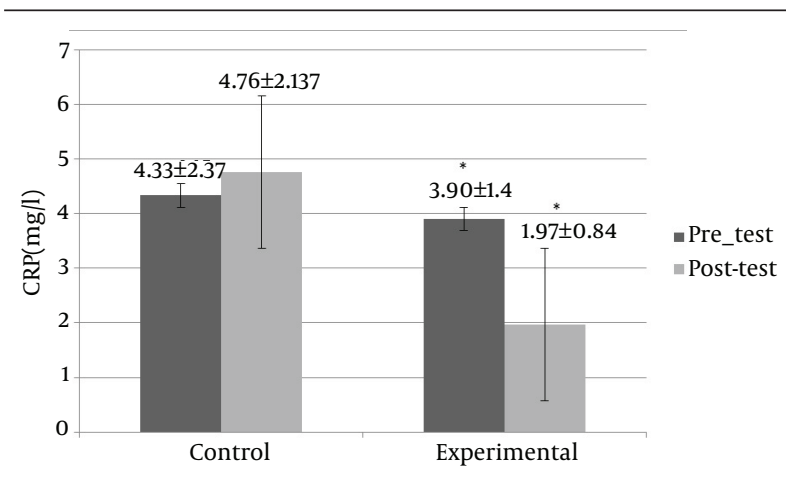

Figure 1. A Comparison Between the Average Changes in C-Reactive Protein $(\mathrm{mg} / \mathrm{L})$ in the Experimental and Control Groups After Eight Weeks of the Rope Skipping Exercises

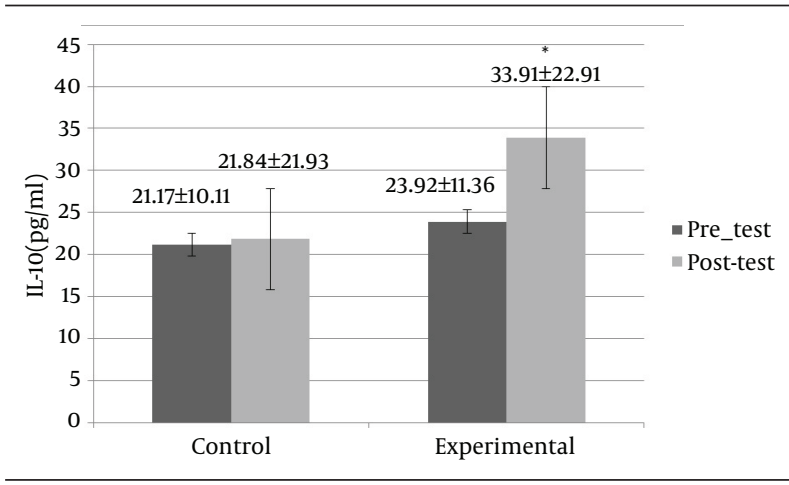

Figure 2. A Comparison Between the Average Changes in the Interleukin-10 (pg/dl) Levels in the Experimental and Control Groups After Eight Weeks of the Rope Skipping Exercises

\section{Discussion}

According to the findings of this study, following eight weeks of the rope skipping exercises the, body weight, body fat percentage, BMI and CRP were significantly decreased and VO2max was significantly increased. Whereas in the comparison between the groups the IL10 showed a nonsignificant increase, in the comparison within the groups, the experimental group showed a significant increase. It is known that physical inactivity is largely involved in the development of the obesity and infection (4). The results of the present study confirm this and indicate the effect of aerobic exercise in reducing body composition. The decrease in body composition may be due to the activity of adipose tissue lipolysis (19). Prolonged exercise increases the rate of lipolysis in adipose tissue. This fact has been confirmed using micro dialysis of extracellular space of subcutaneous adipose tissue. When you exercise, the main activator of lipolysis is a sympathoadrenal system. Using this method it is showed that a mechanism for blocking alpha-adrenergic regulates lipolysis at rest, whereas beta-adrenergic stimulation during exercise is important. The beta-adrenergic effect originates from stimulation of the sympathetic nerves or epinephrine. Epinephrine hormone is considered as a main activator of hormonesensitive lipase. However, there are other hormones that stimulate lipolysis (19). Finally, by increasing the lipolysis activity of adipose tissue the amount of body compounds, including BMI, body fat percentage and weight, decrease (19). Another study demonstrated that eight weeks of intensive aerobic exercise $(35 \%-75 \%$ of maxi- 
Zakavi I et al.

mal heart rate, three days per week for 40 - 50 minutes) reduced weight, body fat percentage, BMI in men with type 2 diabetes. It seems that a more duration of exercise per session as well as moderate intensity of exercise will reduce the body mass, weight, and fat percentage (20). Furthermore, Taghian et al. (21) expressed that 12 weeks of aerobic exercise in 20 obese female students, showed a significant reduction in the weight, fat percentage, and BMI that cause of reduction of body compounds in the investigations depends on the intensity and the duration of the exercise. In addition, Davoodi et al. (22) indicated that exercises with medium intensity $50 \%-70 \%$ of maximum heart rate, a 41-minute exercise, three days a week, for eight weeks) significantly reduce the weight, the percentage of fat, and BMI in obese young girls.

Moreover, aerobic exercise increases significantly oxidative enzymes present in the mitochondria and the increase, in turn, creates circumstances in which the active tissues use more value of oxygen available and thus increase VO2max (21). Most studies have shown a significant increase in VO2max. The results of the studies by Lim et al. (23) have shown a significant increase in VO2max that are consistent with the result of our study. A slow start and gradual progress are one of the principles of aerobic exercise, intensity, duration, type and number of training sessions per week (24) that are consistent with the results of the study, may be a reason for consistency of the findings of the present study with the studies conducted.

Several studies examined the influence of aerobic exercise on the level of serum CRP and mainly the reduction of serum concentrations of CRP. In this study a significant difference was evident between the experimental group before and after eight weeks of the rope skipping exercises compared to the control group.

Martins et al. (25) showed that aerobic and resistance exercise decreases significantly values of CRP. Tchernof et al. (26) said that weight loss alone, without changing physical activity, lowers CRP significantly. Furthermore, Olson et al. (27) reported the reduced levels of CRP after one year of resistance exercise in the obese women. Lakka et al.(17) reported the reduced levels of CRP by exercise, as well. However, the findings of this research are inconsistent with some studies (16, 28-30). Kelley and Kelley have reported that eight weeks of aerobic exercise in obese children did not decrease CRP (28). Hammett et al. (16) and Nassis et al. (29) in their studies reported no difference in CRP levels in obese individuals. The length and intensity of the training period are an important determining factor of change in CRP by the exercise that is a reason for consistency or inconsistency of the results of this study with other studies.

Adipose tissue is an important factor in the amount of CRP. C-reactive protein is one of the factors that are created by the liver in the acute stages of infection. Measurement and evaluation of this protein are a good factor in the study of the progress of infectious diseases or its intensity (31). Investigations have shown that in both genders of obese individuals, CRP level is higher than ordinary people. Obesity creates an inflammatory condition in the body that is associated with increased CRP (6). Interleukins secreted from adipose tissue (IL-6 and TNF- $\alpha$ ) play a role in increasing observed and in the obesity (32). The amounts of CRP in the extreme and heavy sports considerably increase; although it reduces in the regular moderate intensity exercises (31).

The present study showed that after eight weeks of the rope skipping exercises there was not a significant difference between the serum IL-10 levels in the experimental group compared to the control group; however, the serum IL-10 levels in the experimental group were associated with a significant increase; furthermore, development of inflammation in overweight and obese individuals is higher (20). Cytokines are proinflammatory hormones that are involved in regulating the growth and differentiation of function of many cells of the body and play an important role in strengthening the immune response (10). The expression of cytokines on the sport effect is highly dependent on the type, severity, and duration and volume of exercise and training period (33). Among the cytokines, IL-10 is considered as a useful and protecting cytokine for the human metabolism. Because obesity is considered as a proinflammatory state the serum multiple cytokine levels may vary according to the degree of weight loss in obese patients. Interleukin-10 levels increase with weight loss in obese patients along with improved metabolic disorders (11). Also, IL-10 is positively correlated with fitness, so that after an intense and overwhelming exercise, large increase is produced in the amounts of IL-6, which is associated with the increase in IL-10 (anti-inflammatory cytokines) in the hours after exercise (31). Before exercise the IL-10 levels are lower; so, the regular exercise with a moderate intensity reduces proinflammatory cytokines such as IL- 6 and increases anti-inflammatory cytokine such as IL-10 (12). Nunes et al. (34) reported that endurance safety impacts cause a significant increase in serum IL-10 concentrations in blood that is consistent with the results of the present study. Hirose et al. (35) stated that the activities that performed resistive eccentric cause a significant increase in serum IL-10. In addition, Markovitch et al. (14) and Wilund (36) stated that by increasing physical fitness, concentrations of IL-10 increase; thus, IL-10 has a direct relationship with the fitness that is consistent with the results of the present study. In study of Kasapis and Thompson (37) indicated that some of the beneficial effects of physical activity increase the IL-10 by stimulating anti-inflammatory actions. Furthermore, in a study in order to investigate the six-week endurance exercise effect on the levels of IL-10 in tumor tissue of female mouse with breast cancer, Shiri et al. (38) reported that the six-week endurance exercise caused a significant increase of IL-10 compared to the control group that is consistent with the results of the present 
study. The reason for increased IL-10 in these studies depends on intensity of physical activity and fitness levels of people (39) that is consistent with the results of the present study. In a study by Hovanloo et al. (39), no significant difference existed in the serum levels of IL-10 by the 6-session low-volume Sprint Interval Training (SIT) and high-volume continuous endurance training that is inconsistent with the results of the present study. Uchida et al. (40) reported that there was no change in IL-10 in a comparison between the effects of different intensities of the bench press that is inconsistent with the results of the present study. In their study, in order to investigate the water aerobic exercise influence on the IL-10 of multiple sclerosis patients. The water aerobic exercise influence on the IL-10 of multiple sclerosis patients, Bezheh et al. (41) reported that an 8-week water aerobic exercise decreases IL-10 in patients with multiple sclerosis that is inconsistent with the results of the present study. Calle and Fernandez (42) stated that the increase in IL-10 after exercise has been induced by increased production of IL-6, and is a reason for a failure of the increase in some studies is due to low volume of activity; and Neubauer et al. (43) suggested that intensity of exercise is the factor of increased IL-10, which it is a reason for consistency or inconsistency of the results of this study with other studies.

According to the findings of the current study and that the rope skipping is a relatively aerobic and a very lowcost exercise and did not have a need for expensive tools and sports locations, and the distance; so, it increases the anti-inflammatory markers, and reduces the risk of cardiovascular disease. The results obtained from the present study help the physical education trainers to pay attention to the components of the immune system and its relationship with the various sports.

\section{Acknowledgements}

We would like to express our specific thanks to the east of Ahvaz health network management, Mr. Mehrdad Sharifi; vice deputy of training and physical education in the Training and Education Department of Baghmalek County; the management and staff of the Shahid Tabatabaie Hospital of Baghmalek County as well as staff of the Immunology laboratory of the Ahvaz Jundishapur University of Medical Sciences. Furthermore, we would like to thank all of the people who have been involved in this study, especially the respectful participants. The authors declare that there are no conflicts of interest.

\section{Authors' Contributions}

Study concept and design: Iman Zakavi and Mojgan Bani Hashemi Emam Ghaisii. Analysis and interpretation of data: Banafshe Bizhani. Drafting of the manuscript: Iman Zakavi. Critical revision of the manuscript for important intellectual content: Iman Zakavi and Mojgan Bani Hashemi Emam Ghaisii. Statistical analysis: Banafshe Bizhani.

\section{References}

1. Ozcelik O, Celik H, Ayar A, Serhatlioglu S, Kelestimur H. Investigation of the influence of training status on the relationship between the acute exercise and serum leptin levels in obese females. Neuro Endocrinol Lett. 2004;25(5):381-5.

2. Kim ES, Im JA, Kim KC, Park JH, Suh SH, Kang ES, et al. Improved insulin sensitivity and adiponectin level after exercise training in obese Korean youth. Obesity (Silver Spring). 2007;15(12):3023-30.

3. Nascimento H, Rocha S, Rego C, Mansilha HF, Quintanilha A, Santos-Silva A, et al. Leukocyte Count versus C-Reactive Protein Levels in Obese Portuguese Patients Aged 6-12 Years Old. Open Biochem J. 2010;4:72-6.

4. Michishita R, Shono N, Inoue T, Tsuruta T, Node K. Associations of monocytes, neutrophil count, and C-reactive protein with maximal oxygen uptake in overweight women. J Cardiol. 2008;52(3):247-53.

5. Mackinnon LT. Advances in exercise immunology. Hum Kinet;1999.

6. Rosenson RS, McCormick A, Uretz EF. Distribution of blood viscosity values and biochemical correlates in healthy adults. Clin Chem. 1996;42(8 Pt 1):1189-95.

7. Fischer CP, Berntsen A, Perstrup LB, Eskildsen P, Pedersen BK. Plasma levels of interleukin- 6 and C-reactive protein are associated with physical inactivity independent of obesity. Scand J Med Sci Sports. 2007;17(5):580-7.

8. Ruiz JR, Ortega FB, Meusel D, Sjostrom M. Traditional and novel cardiovascular risk factors in school-aged children: A call for the further development of public health strategies with emphasis on fitness.J Pub Health. 2007;15(3):171-7.

9. Lowder T, Padgett DA, Woods JA. Moderate exercise early after influenza virus infection reduces the Th1 inflammatory response in lungs of mice. Exerc Immunol Rev. 2006;12:97-111.

10. Taghavi K, Frnia P, Anooshe S, Bayyat M, Kazem poor M, Masjedi MR. Comparison of Serum TNF-R, Interleukin-10, Interleukin 12 and interferon gammainduced diseaseso fmycobact eriumtubercul osisand non tuberculosis. s. 2010; 18(4):355-360. [In Persian]. Iran J Shaheed Sadoughi Univ Med Sci. 2010;18(4):355-60.

11. Jung SH, Park HS, Kim KS, Choi WH, Ahn CW, Kim BT, et al. Effect of weight loss on some serum cytokines in human obesity: increase in IL-10 after weight loss. J Nutr Biochem. 2008;19(6):371-5.

12. Agha Alinejad H, Molanouri SM. Exercise Induced Release of Cytokines From Skeletal Muscle: Emphasis on IL-6. Iran J Endocrinol Metab. 2010;12(2):181-90.

13. Gleeson M. Immune function in sport and exercise.J Appl Physiol (1985). 2007;103(2):693-9.

14. Markovitch D, Tyrrell RM, Thompson D. Acute moderate-intensity exercise in middle-aged men has neither an anti- nor proinflammatory effect. J Appl Physiol (1985). 2008;105(1):260-5.

15. Selvin E, Paynter NP, Erlinger TP. The effect of weight loss on C-reactive protein: a systematic review. Arch Intern Med. 2007;167(1):31-9.

16. Hammett CJ, Prapavessis H, Baldi JC, Varo N, Schoenbeck U, Ameratunga R, et al. Effects of exercise training on 5 inflammatory markers associated with cardiovascular risk. Am Heart J. 2006;151(2):367 e7-367 e16.

17. Lakka TA, Lakka HM, Rankinen T, Leon AS, Rao DC, Skinner JS, et al. Effect of exercise training on plasma levels of C-reactive protein in healthy adults: the HERITAGE Family Study. Eur Heart J. 2005;26(19):2018-25.

18. Minasyan V, Moradi SM, Mojtahedi H, Ghasem GH. The Evaluation of Health-Related Physical Fitness Status of Men aged between 50 and 65 in Isfahan and Comparison with Available Norms. Bioscie Phys Act. 2013;4(14):111-27.

19. Nicklas BJ, Hsu FC, Brinkley TJ, Church T, Goodpaster BH, Kritchevsky SB, et al. Exercise training and plasma C-reactive protein and interleukin-6 in elderly people. J Am Geriatr Soc. 2008;56(11):2045-52.

20. Shavandi N, Saremi A, Ghorbani A, Parastesh M. Relationship between adiponectin and insulin resistance in type II diabetic men after aerobic training. Arak Med Uni J. 2011;14(2):43-50.

21. Taghian F, Zolfaghary M, Hedayati M. Effect of 12 weeks aerobic exercise on Visfatin level and insulin resistance in obese women. Razi J Med Sci. 2014;20(116):35-44.

Jentashapir J Health Res. 2015;6(4):e24720 
22. Davoodi B, Zilaei Bouri S, Ahangarpor A, Zilaei Bouri M. Effects of two different physical exercises on plasma levels of adiponectin and resistin in obese and overweight young girls. AMUJ. 2014;17(4):27-37.

23. Lim S, Choi SH, Jeong IK, Kim JH, Moon MK, Park KS, et al. Insulinsensitizing effects of exercise on adiponectin and retinol-binding protein-4 concentrations in young and middle-aged women. JClin Endocrinol Metab. 2008;93(6):2263-8.

24. Rawson ES, Freedson PS, Osganian SK, Matthews CE, Reed G, Ockene IS. Body Mass Index, but Not Physical Activity, Is Associated with C-Reactive Protein. Med Sci Sport Exe. 2003;35(7):1160-6.

25. Martins RA, Neves AP, Coelho-Silva MJ, Verissimo MT, Teixeira AM. The effect of aerobic versus strength-based training on highsensitivity C-reactive protein in older adults. Eur J Appl Physiol. 2010;110(1):161-9.

26. Tchernof A, Nolan A, Sites CK, Ades PA, Poehlman ET. Weight loss reduces C-reactive protein levels in obese postmenopausal women. Circulation. 2002;105(5):564-9.

27. Olson TP, Dengel DR, Leon AS, Schmitz KH. Changes in inflammatory biomarkers following one-year of moderate resistance training in overweight women. Int $J$ Obes (Lond). 2007;31(6):996-1003.

28. Kelley GA, Kelley KS. Effects of aerobic exercise on C-reactive protein, body composition, and maximum oxygen consumption in adults: a meta-analysis of randomized controlled trials. Metabolism. 2006;55(11):1500-7.

29. Nassis GP, Papantakou K, Skenderi K, Triandafillopoulou M, Kavouras SA, Yannakoulia M, et al. Aerobic exercise training improves insulin sensitivity without changes in body weight, body fat, adiponectin, and inflammatory markers in overweight and obese girls. Metabolism. 2005;54(11):1472-9.

30. Ferguson MA, White LJ, McCoy S, Kim HW, Petty T, Wilsey J. Plasma adiponectin response to acute exercise in healthy subjects. Eur Appl Physiol. 2004;91(2-3):324-9.

31. Mohammadi HR, Taghian F, Khoshnam MS, Rafati M, Sabagh M. The effect of acute physical exercise on serum IL-6 and CRP levels in healthy non-athlete adolescents. J jahrom univ med sci. 2011;9(2):27-33.
32. Kim JA, Park HS. White blood cell count and abdominal fat distribution in female obese adolescents. Metabolism. 2008;57(10):1375-9.

33. Syu GD, Chen HI, Jen CJ. Differential effects of acute and chronic exercise on human neutrophil functions. Med Sci Sports Exerc. 2012;44(6):1021-7.

34. Nunes RB, Tonetto M, Machado N, Chazan M, Heck TG, Veiga AB, et al. Physical exercise improves plasmatic levels of IL-10, left ventricular end-diastolic pressure, and muscle lipid peroxidation in chronic heart failure rats.J Appl Physiol (1985). 2008;104(6):1641-7.

35. Hirose L, Nosaka K, Newton M, Laveder A, Kano M, Peake J, et al. Changes in inflammatory mediators following eccentric exercise of the elbow flexors. Exerc Immunol Rev. 2004;10:75-90.

36. Wilund KR. Is the anti-inflammatory effect of regular exercise responsible for reduced cardiovascular disease? Clin Sci (Lond). 2007;112(11):543-55.

37. Kasapis C, Thompson PD. The effects of physical activity on serum C-reactive protein and inflammatory markers: a systematic review. J Am Coll Cardiol. 2005;45(10):1563-9.

38. Shiri Y, Agha-Alinejad H, Gharakhanlou R, Amani Shalamzari S Saei MA. Effect of six weeks endurance training on tumor tissue IL-10 cytokine levels in breast cancer bearing mice. Iran J Endocrinol Metab. 2014;16(3):205-10.

39. Hovanloo F, Karimi F, Zar AAS. The effect of exercise with low and high intensity on activities and neutrophils counts.J Hormozgan Univ med sci. 2009;13(4):253-60.

40. Uchida MC, Nosaka K, Ugrinowitsch C, Yamashita A, Martins EJ, Moriscot AS, et al. Effect of bench press exercise intensity on muscle soreness and inflammatory mediators. J Sports Sci. 2009;27(5):499-507.

41. Bezheh N, Soltani M, Khaleghzade H. Effects of aerobic training in water on IL-6 and IL-10 in patients with Multiple Sclerosis. J Evi bas Care. 2014;4(2):63-72.

42. Calle MC, Fernandez ML. Effects of resistance training on the inflammatory response. Nutr Res Pract. 2010;4(4):259-69.

43. Neubauer O, Konig D, Wagner KH. Recovery after an Ironman triathlon: sustained inflammatory responses and muscular stress. EurJ Appl Physiol. 2008;104(3):417-26. 